\title{
Risk factors and relationship between screening periodicity and risk of cervical cancer among nurses and midwives. A cross-sectional study
}

\author{
Selda Yörük', Ayla Açıkgöz", Hülya Türkmen'"', Gül Ergör'v \\ School of Health, Department of Midwifery, Balıkesir University, Cagis Campus, Balıkesir, Turkey
}

IPhD. Assistant Professor, Department of Midwifery, School of Health, Balıkesir University, Cagis Campus, Balıkesir, Turkey.

(D) orcid.org/0000-0003-3840-1996

"PhD. Lecturer, Vocational School of Health Services, Dokuz Eylül University, Izmir, Turkey.

(D) orcid.org/0000-0001-7749-705X

"'PhD. Midwifery Lecturer, Department of Midwifery, School of Health, Balıkesir University, Cagis Campus, Balıkesir, Turkey.

(D) orcid.org/0000-0001-6187-9352

"MD. Professor, Department of Public Health,

Faculty of Medicine, Dokuz Eylül University

Izmir, Turkey.

(D) orcid.org/0000-0002-2263-7526

KEY WORDS:

Nurse midwives.

Uterine cervical neoplasms.

Papanicolaou test.

\begin{abstract}
BACKGROUND: If nurses and midwives undergo cervical cancer screening regularly, they can become role models for other women regarding this screening.

OBJECTIVES: The aims here were (i) to determine factors associated with undergoing cervical cancer screening; and (ii) to examine the association of cervical cancer screening periodicity with cervical cancer risk levels among nurses and midwives.

DESIGN AND SETTING: Cross-sectional study in a public hospital.

METHODS: 466 nurses and midwives participated in this study. The relationships between undergoing Pap smear screening and sociodemographic characteristics, cervical cancer risk factors, perception of cervical cancer risk and calculated cervical cancer risk levels were examined. Cervical cancer risk levels were determined using the "Your Disease Risk" assessment tool (Washington University).

RESULTS: $35 \%$ of the nurses and midwives had undergone Pap smear testing at least once in their lifetimes. The odds of having undergone Pap smear testing were higher among smokers (odds ratio, OR: 2.08; 95\% confidence interval, $\mathrm{Cl}$ : 1.24-3.48) and among those who perceived their risk of cervical cancer to be high (OR: 3.60; 95\% Cl: 1.36-9.51). The frequency of undergoing Pap smear testing at least once in a lifetime was higher among primiparae (OR: 17.99; 95\% Cl: 6.36-50.84) and secundiparae (OR: $41.53 ; 95 \% \mathrm{Cl}: 15.01-114.91)$ than among nulliparae. No relationship was found between Pap smear test periodicity and calculated risk level. CONCLUSION: There is a need to assess motivational barriers that might lead to low levels of Pap smear screening among nurses and midwives who are role models for women regarding cervical cancer prevention.
\end{abstract}

\section{INTRODUCTION}

Cervical cancer screening using Pap smear tests is a cost-effective method for preventing cancer. ${ }^{1,2}$ Diagnosing and treating cervical cancers at the premalignant stage will decrease both incidence and mortality. ${ }^{1}$ In the United States, Finland, Sweden, Denmark and Norway, the incidence of cervical cancer has been significantly reduced through the use of Pap smear test screening. ${ }^{3,4}$

However, in developing countries such as Turkey, the incidence of cancer is gradually increasing. ${ }^{5}$ This increase reveals that there is a need for effective application of cancer screening programs. The American Cancer Society has recommended that all women should start to undergo cervical cancer screening from the age of 21 , and that women aged 30-65 years should undergo Pap smear screening once every three years. ${ }^{6}$ In Turkey, Pap smear test screening is recommended for women aged 30-65 years within the scope of the National Cancer Control Program. ${ }^{7}$ If a person has a high risk of cervical cancer, it is recommended that testing should be done from an early age. ${ }^{4}$

Nurses and midwives work at every stage of the healthcare system and are the professionals who come into direct contact with society. Nurses and midwives' lifestyles relating to cervical cancer and the degree to which they self-apply methods for early diagnosing of this condition are indicative of their individual awareness within this field. Positive attitudes and behavior regarding Pap smear testing among healthcare professionals may contribute towards prevention of cervical cancer both for themselves and for the community. ${ }^{8}$

Despite the role of nurses and midwives in cervical cancer screening, there is little knowledge about Turkish nurses and midwives' own cervical cancer screening behavior. Studies conducted 
among healthcare professionals in Turkey have focused more on the frequency of screening. ${ }^{9-13}$ Only two of these studies examined the factors affecting whether individuals choose to undergo Pap smear tests. It was determined that age affected screening behavior, ${ }^{9}$ and that having information on risk factors did not affect screening behavior. ${ }^{12}$ However, we did not find any studies in which cervical cancer risk levels among midwives and nurses were examined.

\section{OBJECTIVES}

The aims of this study were (i) to determine factors associated with undergoing cervical cancer screening; and (ii) to examine the association of cervical cancer screening periodicity with cervical cancer risk levels among nurses and midwives working in two Turkish public hospitals.

\section{METHODS}

The potential participants for this cross-sectional study comprised female nurses and midwives working in two different hospitals located in the province of Ballkesir in Turkey $(n=680)$. These hospitals are the largest public hospitals in this province, and the highest numbers of nurses and midwives work in these institutions. No sample selection was conducted for this study: the aim was to reach the entire potential population.

Prior to undertaking the study, written permissions were obtained from the Balıkesir University Ethics Committee for NonInterventional Studies and from the managements of the two hospitals. Participants gave their informed consent to be included in this study.

Research data were collected between January 2016 and September 2017. The data were collected through a survey that was created by the present researchers taking into consideration information from the literature within this field. The survey questionnaires were distributed to all midwives and nurses at the institutions where they worked, in closed envelopes. The questionnaire consisted of questions to determine the survey participants' educational background, marital status, cervical cancer risk perception and frequency of undergoing Pap smear tests. It also included questions taken from the "Your Disease Risk" program, ${ }^{14}$ which were used to calculate the level of cervical cancer risk.

There were 202 nurses and midwives who refused to participate in the study. A further 12 midwives and nurses were excluded from the study because they did not complete the survey. Thus, a total of 466 nurses and midwives fully filled out the questionnaire. The response rate was therefore $68.5 \%$.

In order to determine the cervical cancer risk level, the risk assessment tool "Your Disease Risk", published by the Washington University School of Medicine, was used. ${ }^{14}$ In the risk calculation software, assessments were made according to the following features: age; histories of cancer, human immunodeficiency virus
(HIV), hysterectomy, smoking and sexually transmitted diseases (STDs); number of sexual partners; age at the time of initial sexual intercourse; use of condoms; number of children delivered; and whether any Pap smear tests had been undertaken over the last three to five years. The questions asked through this software were added to the questionnaire.

In the risk calculation model for cervical cancer, there were seven risk categories: very much below average, much below average, below average, average, above average, much above average and very much above average. In our study, the cervical cancer risk among the participants was found to be "average", "below average" or "much below average". These three groups were analyzed in the chi-square test. In examining the relationship between risk levels and experience of having undergone Pap smear tests, the cervical cancer risk levels "below average" and "much below average" were combined into a single group, named the "low" group, and in the logistic regression analysis, the assessment was thus made in terms of two groups, named the "average" and "low" groups.

For analytical purposes in the chi-square test, the following variables were dichotomized: marital status (currently married versus unmarried); smoking status (currently smoking versus non-smoker); and having given birth (having given birth versus not having given birth). The following variables were analyzed as three groups: age (20-29 years versus 30 -39 versus $\geq 40$ ); cervical cancer risk perception (high versus low versus unknown); and calculated cervical cancer risk level (average versus below average versus much below average). In the logistic regression analysis, age $(\geq 30$ years versus $<30$ ) and calculated cervical cancer risk level (average versus low) were dichotomized. The number of births was divided into four groups (no births, one birth, two births or three births). The frequency of having Pap smear screening was assessed in four groups (first time, once a year, once every 2-3 years or at irregular intervals) and the latest Pap smear screening was assessed in four groups (within one year, 1-3 years ago, 4-5 years ago or more than five years ago).

The data were analyzed through the Statistical Package for the Social Sciences (SPSS) for Windows 20.0 statistical software. Continuous variables were presented as means and standard deviations; and categorical variables were presented as numbers and percentage distributions. The characteristics of participants who had undergone Pap smear testing once in their lifetimes and those who had not been screened were compared using the chi-square test for categorical variables and using Fisher's exact test for categorical variables with small cell counts. To investigate factors associated with screening for cervical cancer, we used simple and adjusted logistic regression models. The variables that were found to be statistically significant through the chi-square test were included in the logistic regression model. The statistical significance level was taken to be $\mathrm{P}<0.05$. 


\section{RESULTS}

The mean age of the participants was found to be $33.3 \pm 7.4$ (minimum $=22$; maximum $=55$ ) $35.0 \%$ of the participants had undergone Pap smear testing at least once in their lifetimes. The most common reason for not having undergone Pap smear testing was found to be negligence (Table 1).

The characteristics of the participants according to screening status are shown in Table 2. The incidence of having undergone Pap smear testing at least once in a lifetime was higher among subjects who were older, who were smokers, who had given birth, who had histories of STD, who had histories of chronic diseases, who perceived their risk to be high and who had average risk of cervical cancer (Table 2). These significant variables were examined together in the logistic regression model.

The incidence of having undergone Pap smear testing was higher among smokers than among non-smokers (odds ratio, OR: 2.08; 95\% confidence interval, CI: 1.24-3.48) and among those who stated that they perceived their risk of cervical cancer to be high, compared with those who perceived their risk to be low (OR: 3.60; 95\% CI: 1.36-9.51). The frequency of having undergone Pap smear testing at least once in a lifetime was higher among participants who had given birth once (OR: 17.99, 95\% CI: 6.36-50.84) and among participants who had given birth twice (OR: 41.53, 95\% CI: 15.01-114.91) than among those who had never given birth (Table 3 ).

Ninety-four percent of the participants were found to have a low level of cervical cancer risk. No relationships were detected between Pap smear test periodicity and the calculated risk level (Table 4).

\section{DISCUSSION}

This study determined the association between having undergone Pap smear testing and cervical cancer risk levels among female nurses and midwives who were providing services in two hospitals. In this study, about one-third of the participants

Table 1. Proportions of nurses and midwives who had and had not undergone Pap smear testing and reasons for not undergoing any tests

\begin{tabular}{lcc} 
& Number & $\%$ \\
\hline Pap smear test & 163 & 35.0 \\
Done & 303 & 65.0 \\
Not done & 14 & 4.6 \\
Reasons for not having undergone Pap smear testing & \\
Not knowing that it is necessary & 199 & 65.7 \\
Neglect & 17 & 5.6 \\
Afraid of undergoing a smear test & 13 & 4.3 \\
Ashamed of undergoing a smear test & 2 & 0.7 \\
Afraid of cervical cancer & 58 & 19.1 \\
Being single & &
\end{tabular}

had undergone Pap smear testing at least once in their lifetimes. The most common reason for not having undergone Pap smear testing was found to be negligence. The incidence of having undergone Pap smear testing once in a lifetime was higher among smokers. This rate was higher among the individuals who stated that they perceived their risk of cervical cancer

Table 2. Comparison of characteristics between participants who had been screened once in their lifetimes and participants who had not $(n=466)$

\begin{tabular}{|c|c|c|c|c|c|}
\hline \multirow{3}{*}{ Characteristics } & \multicolumn{4}{|c|}{ Pap smear test } & \multirow{3}{*}{$\mathbf{P}$} \\
\hline & \multicolumn{2}{|c|}{ Done } & \multicolumn{2}{|c|}{ Not done } & \\
\hline & $\begin{array}{l}\text { Number } \\
(n=163)\end{array}$ & $\%$ & $\begin{array}{l}\text { Number } \\
(n=303)\end{array}$ & $\%$ & \\
\hline \multicolumn{6}{|l|}{ Age group (years) } \\
\hline $20-29$ & 45 & 27.6 & 123 & 40.6 & \multirow{3}{*}{0.001} \\
\hline $30-39$ & 64 & 39.3 & 152 & 50.2 & \\
\hline$\geq 40$ & 54 & 33.1 & 28 & 9.2 & \\
\hline \multicolumn{6}{|l|}{ Educational status } \\
\hline High school & 25 & 15.3 & 51 & 16.8 & \multirow{3}{*}{0.326} \\
\hline Associate's degree* & 64 & 39.3 & 98 & 32.3 & \\
\hline Bachelor's degree & 74 & 45.4 & 154 & 50.9 & \\
\hline \multicolumn{6}{|l|}{ Marital status } \\
\hline Married & 140 & 85.9 & 183 & 60.4 & \multirow{2}{*}{0.001} \\
\hline Unmarried & 23 & 14.1 & 120 & 39.6 & \\
\hline \multicolumn{6}{|l|}{ Smoking status } \\
\hline Smoker & 65 & 39.9 & 67 & 22.1 & \multirow{2}{*}{0.001} \\
\hline Non-smoker & 98 & 60.1 & 236 & 77.9 & \\
\hline \multicolumn{6}{|l|}{ Childbirth } \\
\hline Having given birth & 155 & 95.1 & 139 & 45.9 & \multirow{2}{*}{0.001} \\
\hline Not having given birth & 8 & 4.9 & 164 & 54.1 & \\
\hline \multicolumn{6}{|l|}{ Live birth count } \\
\hline 1 birth & 38 & 24.5 & 59 & 42.2 & \multirow{3}{*}{0.004} \\
\hline 2 births & 99 & 63.9 & 70 & 50.4 & \\
\hline 3 births & 18 & 11.6 & 10 & 7.2 & \\
\hline \multicolumn{6}{|c|}{ Regular use of condom in sexual intercourse } \\
\hline User & 19 & 11.7 & 27 & 8.9 & \multirow{2}{*}{0.343} \\
\hline Non-user & 144 & 88.3 & 276 & 91.1 & \\
\hline \multicolumn{6}{|l|}{ History of STDs } \\
\hline Present & 7 & 4.3 & 1 & 0.3 & \multirow{2}{*}{0.003} \\
\hline None & 156 & 95.7 & 302 & 99.7 & \\
\hline \multicolumn{6}{|l|}{ History of chronic diseases } \\
\hline Present & 24 & 14.7 & 23 & 7.6 & \multirow{2}{*}{0.015} \\
\hline None & 139 & 85.3 & 280 & 92.4 & \\
\hline
\end{tabular}

Response to the question: "Do you think you have a high risk of cervical cancer?"

$\begin{array}{lccccc}\text { "Yes" } & 38 & 23.3 & 25 & 8.3 & \\ \text { "No" } & 12 & 7.4 & 40 & 13.2 & 0.001 \\ \text { "Don't know" } & 113 & 69.3 & 238 & 78.5 & \end{array}$

Calculated cervical cancer risk

$\begin{array}{lccccc}\text { Average } & 16 & 9.8 & 12 & 4.0 & \\ \text { Below average } & 49 & 30.1 & 60 & 19.8 & 0.001 \\ \text { Much below average } & 98 & 60.1 & 231 & 76.2 & \end{array}$

STD = sexually transmitted disease.

*Associate's degree programs take two years. High school graduates can qualify for associate's degree programs. 
to be high, compared with those who perceived their risk to be low. The frequency of having undergone Pap smear testing at least once in a lifetime was higher among those who had given birth once or twice, compared with those who had never given birth. The majority of the participants had low risk levels.

Table 3. Factors associated with screening for cervical cancer among midwives and nurses $(n=466)^{*}$

\begin{tabular}{|c|c|c|c|}
\hline Variables & $\begin{array}{l}\text { Unadjusted } \\
\text { OR } \\
(95 \% \mathrm{Cl})\end{array}$ & $\begin{array}{l}\text { Adjusted } \\
\text { OR } \\
(95 \% \mathrm{Cl})\end{array}$ & $\mathbf{P}$ \\
\hline $\begin{array}{l}\text { Age group } \\
\qquad \begin{array}{l}\text { Age } \geq 30 \\
\text { Age }<30^{* *}\end{array}\end{array}$ & $\begin{array}{c}1.79+\dagger \\
(1.18-2.72) \\
1.00\end{array}$ & $\begin{array}{c}1.08 \\
(0.65-1.79) \\
1.00\end{array}$ & 0.753 \\
\hline $\begin{array}{l}\text { Marital status } \\
\text { Married } \\
\text { Unmarried }^{* *}\end{array}$ & $\begin{array}{c}3.98+\dagger \\
(2.44-6.65) \\
1.00\end{array}$ & $\begin{array}{c}0.54 \\
(0.22-1.29) \\
1.00\end{array}$ & 0.170 \\
\hline $\begin{array}{l}\text { Smoking status } \\
\text { Smoker } \\
\text { Non-smoker** }\end{array}$ & $\begin{array}{c}2.33+\dagger \\
(1.53-3.53) \\
1.00\end{array}$ & $\begin{array}{c}2.08 \\
(1.24-3.48) \\
1.00\end{array}$ & 0.005 \\
\hline Response to the question:" $D$ & you think you ha & e a high risk of ce & al cancer? \\
\hline $\begin{array}{l}\text { Don't know } \\
\text { No** }^{* *}\end{array}$ & $\begin{array}{c}4.99+\dagger \\
(2.22-11.68) \\
1.58 \\
(0.81-3.24) \\
1.00\end{array}$ & $\begin{array}{c}3.60 \\
(1.36-9.51) \\
1.40 \\
(0.62-3.10) \\
1.00\end{array}$ & 0.010 \\
\hline $\begin{array}{l}\text { Cervical cancer risk level } \\
\text { Average } \\
\text { Low }^{* *}\end{array}$ & $\begin{array}{c}2.63+\dagger \\
(1.20-5.85) \\
1.00\end{array}$ & $\begin{array}{c}1.00 \\
(0.64-0.74) \\
1.00\end{array}$ & 0.999 \\
\hline $\begin{array}{l}\text { Number of births } \\
1 \text { birth }\end{array}$ & $\begin{array}{c}13.06 \\
(5.93-31.47)\end{array}$ & $\begin{array}{c}17.99 \\
(6.36-50.84)\end{array}$ & 0.001 \\
\hline 2 births & $\begin{array}{c}28.67 \\
(13.75-66.34)\end{array}$ & $\begin{array}{c}41.53 \\
(15.01-114.91)\end{array}$ & 0.001 \\
\hline 3 births & $\begin{array}{c}35.37 \\
(12.68-107.02)\end{array}$ & $\begin{array}{c}1.40 \\
(0.23-0.41)\end{array}$ & 0.900 \\
\hline Not having given birth ${ }^{* *}$ & 1.00 & 1.00 & \\
\hline
\end{tabular}

Table 4. Comparison of incidence of Pap smear testing according to cervical cancer risk level $(n=163)$

\begin{tabular}{|c|c|c|c|c|c|}
\hline & \multicolumn{4}{|c|}{ Cervical cancer risk level } & \multirow{3}{*}{$\mathbf{P}^{*}$} \\
\hline & \multicolumn{2}{|c|}{ Average } & \multicolumn{2}{|c|}{ Low } & \\
\hline & $\begin{array}{l}\text { Number } \\
(n=16)\end{array}$ & $\%$ & $\begin{array}{l}\text { Number } \\
(n=147)\end{array}$ & $\%$ & \\
\hline \multicolumn{6}{|c|}{ Frequency of undergoing Pap smear testing } \\
\hline First time & 5 & 31.3 & 34 & 23.1 & \multirow{4}{*}{0.534} \\
\hline Once a year & 1 & 6.2 & 27 & 18.4 & \\
\hline Once every $2-3$ years & 4 & 25.0 & 30 & 20.4 & \\
\hline Irregular intervals & 6 & 37.5 & 56 & 38.1 & \\
\hline \multicolumn{6}{|l|}{ Latest Pap smear screening } \\
\hline Within last year & 7 & 43.8 & 67 & 45.6 & \multirow{4}{*}{0.946} \\
\hline $1-3$ years ago & 4 & 25.0 & 42 & 28.6 & \\
\hline 4-5 years ago & 3 & 18.8 & 13 & 8.8 & \\
\hline More than 5 years ago & 2 & 12.5 & 25 & 17.0 & \\
\hline
\end{tabular}

*Fisher exact test.
No relationships were found between Pap smear test periodicity and the calculated risk level.

Thirty-five percent of the nurses and midwives who participated in this study had undergone Pap smear testing at least once in their lifetimes. The frequency of having undergone Pap smear testing at least once in a lifetime was found to range from $10.2 \%$ to $83.7 \%$ in studies conducted in Turkey and Serbia among women. ${ }^{15,16}$ This rate was found to range from $23.7 \%$ to $45.2 \%$ among healthcare professionals in Turkey. ${ }^{9-13}$ In studies conducted among nurses in different countries, the incidence of Pap smear testing at least once in their lifetimes ranged from $3.4 \%$ to $72.6 \%{ }^{17-22}$ The frequency of having undergone Pap smear testing was higher in our study than in some studies ${ }^{17-20}$ and lower than in other studies. ${ }^{21,22}$ In addition to factors such as age, education and presence of a screening program in a given country, it has been shown that the level of development of countries also has an effect in relation to cervical cancer screening. ${ }^{8}$ Studies in which the incidence of Pap smear testing was lower than in our study were conducted in underdeveloped or developing countries. ${ }^{17-20}$

About two-thirds of the nurses and midwives who participated in this study stated that they had never undergone Pap smear testing. In this study, it was determined that for most of the nurses and midwives who had not undergone Pap smear testing, this was due to their own negligence (65.7\%), according to their responses to the questionnaire. In other studies among nurses and midwives, the most common reasons that they stated for not having undergone such tests were factors such as not considering themselves to be at risk, ${ }^{18,19}$ thinking that screening was not necessary for themselves ${ }^{11,23}$ and negligence. ${ }^{13}$ Encouragement by the partner, regular examination by a gynecologist and suggesting a Pap smear test by these experts are effective measures in increasing the regular undertaking of the Pap smear test. ${ }^{22,24}$ In-service training can be given to nurses and midwives regarding the importance of cervical cancer risk factors and of undergoing Pap smear tests.

In the present study, it was found that the incidence of undergoing Pap smear testing was higher among smokers. Higher levels of carcinogenic substances were found in the cervical mucus of smokers than in the mucus of non-smokers. It is thought that these substances may be effective in aiding development of cervical cancer through causing damage to the deoxyribonucleic acid (DNA) of cervical cells. ${ }^{4}$ In some studies conducted among healthcare professionals, the levels of information regarding the fact that smoking is a risk factor for cervical cancer were found to be low. ${ }^{11,13}$ On the other hand, in other studies, although these levels were found to be high, only low levels of screening were occurring. ${ }^{12,23}$ In our study, the odds of undergoing Pap smear testing were higher among smokers. This significant finding may have been caused by the fact that because of their profession, the research group of this study knew about the fact that smoking is an important risk factor for cervical cancer. 
In the study group, the odds of having undergone Pap smear testing were higher among participants who perceived themselves to be at risk in terms of cervical cancer. Women's perceptions of risk relating to cervical cancer are an important subjective finding: this perception leads them to maintain a healthy lifestyle and take advantage of early diagnostic methods. Perception of high risk of cervical cancer leads women to increase their frequency of undergoing screening. ${ }^{25} 13.5 \%$ of the nurses and midwives who participated in the present study perceived their own risk of cervical cancer as high. In a study conducted among nurses working in a hospital in Turkey, $31.8 \%$ of them indicated that they perceived themselves to be in the at-risk group in terms of cervical cancer. ${ }^{13}$ The proportion of nurses in a study conducted in Singapore who perceived that they had a high level of individual cervical cancer risk was lower than what was found in the present study. ${ }^{22}$ In a study conducted in a group undergoing treatment for high-grade cervical intraepithelial neoplasia, it was found that $64 \%$ of these women perceived themselves to be at high risk and $30 \%$ at low risk. ${ }^{26}$

Multiparity has been linked with higher risk of cervical cancer in women. ${ }^{2}$ In the present study, the frequency of having undergone Pap smear testing at least once in a lifetime was higher among participants who had given birth once or twice than among those who had never given birth. In a study conducted among nurses in India, it was also found that multiparity increased the frequency of Pap smear testing. ${ }^{27}$ However, no relationship was found between the frequency of having undergone Pap smear testing and the number of births, in studies conducted in the general population in India. ${ }^{25,28}$ In the present study, in univariate analysis (chi-square test), it was determined that the incidence of having undergone Pap smear testing increased significantly with increasing age among the nurses and midwives. However, this relationship was not observed in the logistic regression analysis. Among nurses, the incidence of having undergone Pap smear testing was found to be highest in the 40 to 44-year age group in Australia, ${ }^{21}$ in the 35 to 49 -year age group in Singapore $^{22}$ and in the group aged 40 years and over in Turkey. ${ }^{9}$

It is quite difficult to calculate the risk of cervical cancer in absolute terms. However, calculating the individual risk of cervical cancer using the risk calculation models that have been developed may provide a guide in terms of both cervical cancer prevention and early diagnosis. ${ }^{29}$ Nevertheless, it is important to accurately compile risk factors from women in determining the risks. In the present study, the calculated level of cervical cancer risk was low in $94 \%$ of the nurses and midwives, while only $6 \%$ of them had an average level of risk. In a study conducted among women aged 35-69 years, it was calculated that $8 \%$ of them had a high level of cervical cancer risk, while $22 \%$ of them had an average level of risk and $70 \%$ of them had a low level of risk. ${ }^{15}$ In that study, the higher level of risk found among the participants may have resulted from the older age of the participants in that study, compared with those of the present study, along with the higher level of risky behavior among the participants in that study. The low risk presented by the majority of the present study group was due to its young age, with relatively few risky behavioral characteristics. The number of women undergoing treatment for STDs was minimal in the present study.

The risky behavior among the participants that needed to be taken into account in calculating the cervical cancer risks originated from smoking and failure to undergo Pap smear testing over the past three years. About a third of the women in the present study continued to smoke. Açıgöz et al. found that the incidence of undergoing Pap smear testing decreased as the level of cervical cancer risk increased. ${ }^{15}$ However, in the present study, in the univariate analysis (chi-square test), as the level of cervical cancer risk increased, the incidence of having undergone Pap smear testing increased significantly. On the other hand, this relationship was not observed in the logistic regression analysis. Regardless of which part of society women belong to or at which age they are, it needs to be ensured that they can undergo regular Pap smear testing and that their risky behavior is reduced.

The strengths of the present study include the fact that, although several knowledge-attitude-behavior studies on cervical cancer among nurses and midwives in Turkey were found to be available through our review of the literature, there were none in which the level of cervical cancer risk was determined among nurses and midwives. For the first time in Turkey, nurses and midwives' cervical cancer risk levels were identified using risk level determination software.

The limitations of our study relate mainly to data collection. We collected self-reported data. Although self-reporting is an acceptable method of data collection in public health studies, it is associated with the potential for recall bias. Therefore, the results regarding the women's risk levels may have been determined in different manners. The results from this study cannot be generalized to all nurses and midwives. Since the participants did not respond to any question regarding how many partners they had had, the cervical cancer risk level among these individuals may have been lower than the true level because they were considered to be monogamous.

It is undeniable that healthcare professionals have a role to play in raising awareness about cervical cancer within the community. It is necessary to conduct studies aimed at ascertaining what the motivational barriers are that lead to such low levels of Pap smear test screening among nurses and midwives who are role models for women regarding prevention of cervical cancer. Correct information about cervical cancer risk factors and the importance of Pap smear test screening is needed not only for women in the at-risk group but also for nurses and midwives. In this way, socially more effective results regarding prevention of cancer and use of early diagnostic and treatment methods will be obtained. 


\section{CONCLUSION}

It was found that the incidence of having undergone Pap smear testing at least once in a lifetime among nurses and midwives was low in this study. Smokers, participants with a high perception of cervical cancer risk and those who had given birth presented higher levels of having undergone Pap smear testing at least once. Most of the nurses and midwives presented low cervical cancer risk levels. No relationships were found between Pap smear test periodicity and the calculated cervical cancer risk level.

\section{REFERENCES}

1. World Health Organization. Guide to Cancer Early Diagnosis. Geneva: World Health Organization; 2017. ISBN: 978-92-4-151194-0.

2. National Cancer Institute. Cervical Cancer Screening. Available from: https://www.cancer.gov/types/cervical/hp/cervical-screening-pdq. Accessed in 2018 (Jul 16)

3. World Health Organization. Cancer Control: Knowledge into Action: WHO Guide for Effective Programmes: Module 3: Early Detection. Available from: https://www.ncbi.nlm.nih.gov/pubmed/24716262. Accessed in 2018 (Jul 18).

4. IARC Handbooks of Cancer Prevention: volume 10. Cervix Cancer Screening. Lyon: IARC Press; 2005. ISBN-13: 978-92-832-3010-6. Available from: http://www.iarc.fr/en/publications/pdfs-online/prev/ handbook10/HANDBOOK10.pdf. Accessed in 2018 (Jul 16).

5. International Agency for Research on Cancer. GLOBOCAN 2012: Estimated Cancer Incidence, Mortality and Prevalence Worldwide in 2012. Available from: http://globocan.iarc.fr/Pages/online.aspx. Accessed in 2018 (Jul 16).

6. American Cancer Society: Cervical Cancer. The American Cancer Society Guidelines for the Prevention and Early Detection of Cervical Cancer. Available from: https://www.cancer.org/cancer/cervical-cancer/ prevention-and-early-detection/cervical-cancer-screening-guidelines. html. Accessed in 2018 (Jul 16).

7. T. C. Ministry of Health Public Health Agency of Turkey. Turkey Cancer Control Programme. Ministry of Health Publications. Ankara, 2016. Available from: https://www.iccp-portal.org/system/files/plans/Turkiye_ Kanser_Kontrol_Program_English.pdf. Accessed in 2018 (Jul 16).

8. Musa J, Achenbach CJ, O'Dwyer LC, et al. Effect of cervical cancer education and provider recommendation for screening on screening rates: A systematic review and meta-analysis. PLoS One. 2017;12(9):e0183924. PMID: 28873092; doi: 10.1371/journal. pone.0183924.

9. Özçam H, Çimen G, Uzunçakmak C, et al. Evaluation of the Knowledge, Attitude, and Behavior of Female Health Workers about Breast Cancer, Cervical Cancer, and Routine Screening Tests. İstanbul Med J. 2014;15:154-60. doi :10.5152/imj.2014.86548.

10. Can H, Erdem E, Oztekin C, et al. Are primary health care workers aware of cervical cancer risk? Asian Pac J Cancer Prev. 2014;15(16):6669-71. PMID: 25169506.
11. Göl I, Ekin Ö. Knowledge and practices of nurses on cervical cancer, HPV and HPV vaccine in Cankiri state hospital, Turkey. J Pak Med Assoc. 2016;66(12):1621-6. PMID: 28179702.

12. Coskun S, Can H, Turan S. Knowledge about cervical cancer risk factors and pap smear testing behavior among female primary health care workers: a study from south turkey. Asian Pac J Cancer Prev. 2013;14(11):6389-92. PMID: 24377538.

13. Özdemir Ö, Bilgili N. Knowledge and Practices of Nurses Working in an Education Hospital on Early Diagnosis of Breast and Cervix Cancers. TAF Prev Med Bull. 2010;9(6):605-12. doi: 10.5455/ pmb.20100427030359.

14. Washington University School of Medicine. Your Disease Risk. Cervical cancer. Available from: https://siteman.wustl.edu/prevention/ydr/? ScreenControl=YDRGeneral\&ScreenName=YDRAbout. Accessed in 2018 (Jul 16)

15. Açıkgöz A, Ergör G. Cervical cancer risk levels in turkey and compliance to the national cervical cancer screening standard. Asian Pacific J Cancer Prev. 2011;12(4):923-7. PMID: 21790227.

16. Antic LG, Djikanovic BS, Antic DZ, Aleksopulos HG, Trajkovic GZ. Differences in the level of knowledge on cervical cancer among health care students, midwives and patients in Serbia. Asian Pac J Cancer Prev. 2014;15(7):3011-5. PMID: 24815439.

17. Jain SM, Bagde MN, Bagde ND. Awareness of cervical cancer and Pap smear among nursing staff at a rural tertiary care hospital in Central India. Indian J Cancer. 2016;53(1):63-6. PMID: 27146744; doi: 10.4103/0019509X.180823.

18. Unang I, Abasiattai AM, Udoma EJ. Awareness and Practice of Cervical Smear as A Screening Procedure for Cervical Cancer among Female Nurses in A Tertiary Hospital in South-South Nigeria. TAF Prev Med Bull. 2011;10(6):675-80. doi: 10.5455/pmb.20110823055210.

19. Rahman H, Kar S. Knowledge, attitudes and practice toward cervical cancer screening among Sikkimese nursing staff in India. Indian J Med Paediatr Oncol. 2015;36(2):105-10. PMID: 26157287; doi: 10.4103/09715851.158840.

20. Adebara IO, Olufolake AO, Adewara OE, et al. Utilization of pap smear in the prevention of cervical cancer among female health workers at a Nigerian Tertiary Health Facility. Nigerian J of Family Practice. 2007;8(4):41-7. Available from: https://www.ajol.info/index.php/njfp/ article/view/157348. Accessed in 2018 (Jul 16)

21. Nicholls R, Perry L, Gallagher L, et al. The personal cancer screening behaviours of nurses and midwives. J Adv Nurs. 2017;73(6):1403-20. PMID: 27878855; doi: 10.1111/jan.13221.

22. Tay K, Tay SK, Tesalona KC, et al. Factors affecting the uptake of cervical cancer screening among nurses in Singapore. Int J Gynaecol Obstet. 2015;130(3):230-4. PMID:26032624; doi: 10.1016/j.jjgo.2015.03.037.

23. Kress CM, Sharling L, Owen-Smith AA, et al. Knowledge, attitudes, and practices regarding cervical cancer and screening among Ethiopian health care workers. Int J Womens Health. 2015;7:765-72. PMID: 26261427; doi: 10.2147/IJWH.S85138. 
24. Lee SY. Cultural Factors Associated with Breast and Cervical Cancer Screening in Korean American Women in the US: An Integrative Literature Review. Asian Nurs Res (Korean Soc Nurs Sci). 2015;9(2):81-90. PMID: 26160234; doi: 10.1016/j.anr.2015.05.003.

25. Gu C, Chen WT, Zhang Q, et al. Exploring Chinese Women's Perception of Cervical Cancer Risk as It Impacts Screening Behavior: A Qualitative Study. Cancer Nurs. 2017;40(4):E17-E25. PMID: 27281034; doi: 10.1097/ NCC.0000000000000400

26. Andersson S, Belkić K, Demirbüker SS, Mints M, Östensson E. Perceived cervical cancer risk among women treated for high-grade cervical intraepithelial neoplasia:The importance of specific knowledge. PLoS One. 2017;12(12):e0190156. PMID: 29272293; doi: 10.1371/journal. pone.0190156.

27. Shekhar S, Sharma C, Thakur S, Raina N. Cervical Cancer Screening: Knowledge, Attitude and Practices among Nursing Staff in a Tertiary Level Teaching Institution of Rural India. Asian Pac J Cancer Prev, 2013;14 (6):3641-3645. PMID: 23886159; doi: 10.7314/APJCP.2013.14.6.3641.

28. Hyacinth HI, Adekeye OA, Ibeh JN, Osoba T. Cervical cancer and Pap smear awareness and utilization of Pap smear test among Federal civil servants in North Central Nigeria. PLoS One. 2012;7(10):e46583. PMID: 23049708; doi: 10.1371/journal.pone.0046583.

29. Bosch FX, de Sanjosé S. Chapter 1: Human papillomavirus and cervical cancer--burden and assessment of causality. J Natl Cancer Inst Monogr. 2003;(31):3-13. PMID: 12807939.

\section{Sources of funding: None}

Conflict of interest: None

Date of first submission: July 18, 2018

Last received: January 11, 2019

Accepted: January 22, 2019

\section{Address for correspondence:}

Selda Yörük

Department of Midwifery, School of Health, Balıkesir Üniversitesi, Cagis

Campus, 10145 Balıkesir, Turkey

Tel. +902662440010

Fax: +902662497125

E-mail: syoruk@balikesir.edu.tr 